\title{
A Study on Factors Contributing to Communities not Accessing Anti Retro Viral Therapy [ARVs] in some Rural Health Facilities in Eastern Province of Zambia
}

\author{
Article by James Mwale \\ Master of Public Health, Texila American University, Zambia \\ E-mail: jamesamwale2005@yahoo.co.uk
}

\begin{abstract}
The competing economical, social and political challenges makes health to remain neglected in developing countries.

Patients cannot access the ARVs without CD4 count tests, as it is a marker for immune system.

Patients have easy access for HIV (Human Immune Virus) testing than for CD4 count. It is for this reason that Pima CD4 testing machines were introduced to enable those previously unable to offer CD4 testing, to do so.

Study data shows that facilities with Pima CD4 machines have higher patients number on ARVs than those who do not have.

It is for this reason that government and cooperating partners should support procuring more Pima CD4 machine for point of care.
\end{abstract}

\section{Introduction}

The Pima CD4 test is the first true point of care solution for CD4 testing. CD stands for cluster of Differentiation and refers to certain proteins on the surface of blood cells. Cells with different function express different classes of CD protein. In case of CD4 T Lymphocytes, the two determining CD proteins are CD3 and CD4.The body's immune response is co-ordinated by CD4 T Lymphocytes which circulate through the blood. These cells are also known as Thelper cells or Th cells.

After a primary infection with HIV, the virus directly attack CD4 T Lymphocytes to their destruction while using them as host cells for replication. Billions of CD4 T Lymphocytes may be destroyed every day, eventually overwhelming the immune system' s regenerative capacity. Following an acute HIV infection, patients may remain free of HIV related illnesses, often for years, despite on going HIV replication. During this period, the immune system is continuously weakened but remains sufficiently competent to provide immune surveillance and to prevent most infections. Eventually, the progressive loss of CD4 T Lymphocytes results in the breakdown of appropriate immune response and the outbreak of opportunistic diseases, the pathological state of AIDS.

Although the decrease in the total number of $\mathrm{T}$ Lymphocytes may not always exactly match the qualitative functions of the immune system, the total CD4 T Lymphocyte number still remains the most robust surrogate marker of immune competence.

The main objective of Pima CD4 machine is to increase accessibility for early treatment or ARVs. The machine can be used both urban and rural areas. Pima CD4 machine uses battery, solar or alternate current.

However the Pima is designed equally to suit the needs of the health care professional in the field or in the Laboratory the Pima CD4 test provides an affordable, effective and valuable tool in the management of HIV patients.

\section{Background}

Eastern Province is one of the ten provinces of Zambia situated to eastern part of Zambia. The province is surrounded by nine districts, namely: Chipata, Chadiza, Lundazi, Katete, Mambwe, Nyimba, Sinda, Petauke and Vubwi. 
Texila International Journal of Public Health

Volume 4, Issue 4, Dec 2016

The beginning of Anti retro viral therapy (ARVs) started in Chipata district which is the provincial town of the province. This meant that all the districts were to send samples for CD4 count tests to the provincial laboratories at Chipata General Hospital by then, now it is Chipata Central Hospital.

There was overload of work to do CD4 counts for the all the districts with little human resource .Results were coming after one to two months, this meant that some clients found them in critical conditions and others died. Point of care was not their. Until 2002 few CD4 machines where introduced in some districts.2013 where the introduction of Pima CD4 count machine to some health facilities in the districts to improve the turn around time of diagnosis and treatment

\section{Study problem statement}

The factors contributing communities not accessing Anti retro viral therapy services in some rural health facilities in eastern province of Zambia.

There is need to procure more Pima CD4 lymphocytes count machine as it is an effective way of monitoring the status of HIV positive patient as they commence Anti retro viral therapy.

Out of 100 health facilities sampled only 30 represented having Pima CD4 lymphocytes count this is 30\%.Also those have access to ARVs when CD4 count is done and 70\% health facilities refer their clients to other institution and commencement of treatment is compromised because of long distance coverage. Here results may get one or three months before the patients receives them or may get lost on the way.

Records shows that facilities with Pima CD4 count have more clients on HIV tests as well as those taking treatment(ARVs).In comparison with health facilities having no Pima CD4 lymphocyte count machine.

Therefore there is need for the government and cooperating partners to procure more Pima CD4 lymphocytes count machines so that facilities who do not have can benefit from good turnaround time of diagnosis and treatment.

\section{Specific objective}

- Improve turn around time on diagnosis and treatment.

- Increase access on counseling and testing for human immune virus [HIV].

- Increase on openings of ART centres or treatment centres.

\section{Justification for the study}

Patients who only test for HIV have no access to ARVs until a CD4 count test is done then they have access for ARVs, therefore there is need for Pima CD4 test machines to be procured in many health facilities as it helps in monitoring treatment in rural communities as there is no advanced machines.

\section{Literature review}

The Pima CD4 test comprises of a disposable Pima CD4 test cartridge and the Pima analyser, and enables the determination of absolute counts of CD4 T Lymphocytes in whole blood without prior sample preparation.

The disposable cartridge is equipped with means to take up approminately $25 \mathrm{ul}$ of sample and contains dried reagents needed to perform the test. The test is performed in its entirely within the confinement of the cartridge and no part of the analyser has at any time contact with the sample. This minimizes the risk of Analyser contamination and eliminates sample carry over between measurements.

After insertion of the test cartridge in the analyser peristaltic movement first transports the sample into the incubation compartment where the sample interacts with the specific antibodies to immunolabel CD4 T Lymphocytes with two different fluorescent dyes emitting light at two different wavelengths. One antibody is an antibody conjugated to PE- Cy5. The 
second antibody is an anti- CD4 antibody conjugated to PE. After a defined incubation time the stained sample is automatically transferred into the separate reading compartment of the cartridge.

The Pima analyser resembles a fluorescent microscope, equipped with miniaturised optics that detects fluorescence signals, controlled by an embedded computer. CD4 T Lymphocytes carry both CD3 and CD4 surface antigens and therefore emit light at wave lengths specific for both antibody - dye conjugates. This allows the specific differentiation of CD4 T Lymphocytes from other blood cell types carrying only one of the two surface antigens. Specific requirements with regard to signal intensity, object size and many other parameters need to be met in order for an object to be counted as a CD4 T Lymphocyte. This information is encoded in the barcode of every cartridge and is used by the Pima analyser to translate the raw count into a test result of cells/ul. The result is displayed on the analyser' s screen.

Results and the date/time the test was carried out are also stored in an on -board archive and are assigned to a sample ID that has been entered into analyser by the operator. An external Pima printer can be used to print a Pima test report for each test. Data can be retrieved and down loaded by the operator at any time after the test.

It has been noticed that patients who go for early diagnosis of HIV and CD4 testing have advantages of treatment in time. Early treatment help minimize opportunistic infections. Because of this, consideration of procuring and installation of Pima CD4 machines in needy areas must be a priority to the government and cooperating partners. When these services can reach rural hard to reach areas we may prolong lives to those affected.

The beauty of Pima machine, is portable and use both batteries and alternate current. The designed equally suit the needs of the health care professional in the field and non health professionals. In both developed and developing countries Pima does a recommendable work. This machine once charged it can be used for eight hours. Reagents and controls can be stored at room temperatures between 2- 30 degrees centigrade. Samples, EDTA bottles are used or finger prick method.

When it comes to errors, an error code will be shown on the Pima screen for easy maintenance .Pima has also modem and simcard for sending data to centre place for analysis.

\section{The feature of PIMA analyser}

- Has on board data archive

- Embedded software

- No external calibration

- Main A/C and battery power

- Portable and robust

- Absolute CD4 count in 20 minutes.

- Two USB ports - Pima printer and data export.

\section{Reagents- Pima CD4 cartridge}

- All reagents are sealed in the disposable cartridge

- Require only 25ul of capillary or venous whole blood

- No cold chain storage

- No manual sample handling or processing.

\section{Storage}

- Reagents and beads standard are stored at room temperature $2-30$ degrees

- If refrigerated bring to room temperature before use.

- Keep out of direct sunlight

- Open immediately before use only

- The Pima analyser will not run on expired cartridge cassette or Pima beads standards. 
Texila International Journal of Public Health

Volume 4, Issue 4, Dec 2016

\section{PIMA beads standards}

- External standard for daily quality control

- Two ready to use cartilage - Normal , Low

- Have set amounts of fluorescent beads inside each cartridge - No further reagents are required.

- Results should be within ranges specified inside storage box

- Once opened can be used for 6 months.

- Store in storage box at room temperature out of direct sunlight.

\section{Before introduction of Pima cd4 machine in health facilities}

- Patient used to cover long distance to access CD4 count tests

- Results took one to three month's time to be out because of backlog.

- Most patients die before getting the results and treatment

- There was long turnaround time.

- Voluntary counseling and testing for HIV was low because they had no access for CD4 count test and treatment

\section{After introduction of Pima cd4 machine in health facilities}

- Reduced covering long distance

- Improved turnaround time as patients could receive result same day.

- People's health lives improved due to early diagnosis and treatment

- Improved counseling and testing

- Increase demands of services

\section{Methodology.}

This topic explains and examines how data were collected using well developed tested tools as means towards realizing study objectives. In general the topic focuses on the study design, study area, study population, sample size and sampling procedure, data collection tools, data quality control and handling, data analysis, dissemination of results.

\section{Research design}

The study used a cross-sectional design. Data collection methods included questionnaire, record review and observations. The questionnaire was developed by the researcher and was self-administered to the participants.

\section{Research site}

The study site will be Eastern province which is in the eastern part of Lusaka the capital city of Zambia and has a distance of $600 \mathrm{~km}$ from the city. And Chipata is the provincial capital for eastern province. The province has nine districts namely; Nyimba, Petauke, Katete, Chipata, Chadiza, Sinda,Lundazi, Mambwe and Vubwi with approximately a population of 1.8 million.

\section{Study population}

The province has roughly a population of 1.8 million which is inclusive of farmers,business persons,workers in government and non governmental organization and non employed class. The participants in the study will be drawn from all these categories so that there is equal representation of the views.

\section{Sample size and sampling process}

The study sample size from the selection at the province was considered at following the formula and calculation as shown below:

The estimated number of patients accessing the ARVs services after HIV positive and CD4 testing is about 2000 . 
The approach used was for Systematic sampling.

- A complete list of all individuals in the study population was obtained

- $\quad$ Required sample size was determined

- The first individual was selected randomly from the study population list.

- After that each n(number) individual in the study population is selected e.g.

Study population $=500000$

Required sample size $=2000$

Say the first randomly selected number $=120$ from there on wards, select

$500000 / 2000=250^{\text {th }}$ number

Thus, the second number will be $=120+250=370$

The third selected number $=370+250=620$ etc.

It is simple, cheap, quick and convenient to execute if a complete list of the study population is readily available.

\section{Sampling process}

Sampling is the procedure a researcher uses together, people, places or things to study. It is process of selecting a number of individuals or objects from a population such that the selected group contains elements representative of the characteristic found in the entire group Orodho and Komba (2002,Quotd in Kombo and Tromp,2014,P,77).Therefore, this study employed a purpose sampling process/procedure to enable all patients have equal chance of being interview. Selection of the participants was from all categories of patient from health facilities. This technique was used in order to have a generalized representation of the population for the province

\section{Research instruments/ tools.}

The research instruments used in this research study were semi-structured questionnaire with both open and closed ended questions, checklist for materials required for Pima CD4 counting testing and observations. The questionnaire was designed focusing on the specific objectives of the study from which questions were developed. The observation technique was further used to have a physical check whether Pima CD4 count machine were available or not and correctly used in all targeted areas of the health facilities for this study.

\section{Data collection procedure.}

The procedure in which data was collected from the participants was through distribution of the self-administered questionnaire, analysis of the implementation of planned activities from the action plans and budgets for 2016 respectively on Pima CD4 lymphocytes count management. Furthermore, structured type of observations had to be carried out in wards and outpatient department. The reason was to observe whether all HIV positive persons have access to CD4 count as well as Antiretroviral therapy. The interviews were carried out in the months of May and August, 2016. The main questions was factors contributing to communities not accessing Antiretroviral therapy in some rural health facilities in Eastern Province of Zambia.

\section{Data management and analysis}

The raw data collected from participants at the facility had to be checked by the principle researcher each time it was collected and whatever was not convincing, effort was made to ensure correct data was got from all the respondents. This was to ensure completeness and consistence of the data in all the interview guides. The analysis of the data after compilation was through the use of the SPSS (version 20). Data cleaning was performed by running each variable to check the accuracy, inconsistency and missed values.

\section{Pre-testing of tools}

The data collection tools were the semi structured interviews (self-administered questionnaire) and checklist tested in 2 units of the health facilities in order to validate them 
Texila International Journal of Public Health

Volume 4, Issue 4, Dec 2016

prior to roll out to others. As for the observations, these were strictly the responsibility of the researcher as the units were being visited.

Installation of Pima CD4 machines in needy areas must be a priority to the government and cooperating partners. When these services can reach rural communities we may improve community lives

\section{Management of the project}

During this project of Pima CD4 machine count testing, we shall collect data to facilities were Pima CD4 count testing is done. And also collect data in health facilities where there is no Pima machine. While doing this will collect data for voluntary counseling and testing for HIV in health facility.

In this research we shall involve Laboratory scientist, Nurses, Clinical officers, Doctors, Pharmatist and nutritionalist.

\section{Financing}

\begin{tabular}{|l|l|l|l|}
\hline ITEM & QUANTITY & $\begin{array}{l}\text { UNIT PRICE. } \\
\text { USD }\end{array}$ & TOTAL. USD \\
\hline Stationary & & & \\
\hline Leaf pad & 3pieces & 5 & 15 \\
\hline Ream of papers & 2 & 5 & 10 \\
\hline Pens & 10 & 0.15 & 1.5 \\
\hline Printing material & 1000 & 0.3 & 300 \\
\hline $\begin{array}{l}\text { Travel Eastern } \\
\text { province( to and fro) }\end{array}$ & 10 trips & 200 & 2000 \\
\hline $\begin{array}{l}\text { Personnel(research } \\
\text { assistant) }\end{array}$ & $\begin{array}{l}2 \text { personnel's x10 } \\
\text { days }\end{array}$ & $100 \times 2 \times 10$ & 40,000 \\
\hline Pima Machines & 100 & 5000 & 500,000 \\
\hline Reagents Cartridges & $1000[1 \times 100]$ & 200 & 200,000 \\
\hline $\begin{array}{l}\text { Controls Beads } \\
\text { Standard }\end{array}$ & 200 & 100 & 20,000 \\
\hline \multicolumn{2}{|l|}{} & $\begin{array}{l}\text { GRAND } \\
\text { TOTAL }\end{array}$ & $\mathbf{7 6 2 , 3 2 6 . 5 0}$ \\
\hline
\end{tabular}

\section{Time frame}

The project will start with data collection starting May to August.Then September to October,2016. Will be compilation of data and analysis. November,2016 final report writing.

\section{Ethical consideration}

Ethics is defined by Webster's dictory in Bahttachrjee(2012) as conformance to the standards of conducts of given profession or group. He further contents that ethics is the moral distinction between right and wrong and what is unethical may not necessarily be illegal.This is an important aspect in research as it protects participants from harm. To this effect, ethical clearance for the study to commence was granted by provincial Medical Officer, who is the in charge of the province as the province does not have the ethics committee which is supposed to carry out the functions. In addition consent was sought from various health facilities and individual participants before the planned tests could be used to the target group. It was imperative that respondents understood what their participantion in the study would involve. Information about the study as well as the consent forms had to be written and explained and signed consent forms were obtained from each of the respondents. 


\section{Voluntry Participation}

The respondents were informed that participation in the study was voluntary. The purpose of the study was explained to the respondents so that they could make informed decisions about whether or not to participate in the interview. They were assured that participation was purely voluntary and that even if they decided to stop at any time during data collection process, they would not be prejudiced in any way.

\section{Shared confidentiality}

The respondents were informed about shared confidentiality between the researcher and the Texila American University. They were also assured that no information given would be shared with anyone with access to the research findings unless where the respondent gave permission. They were also assured that the information given would remain confidential and they will be no detrimental consequences from the answers given.

\section{Informed consent}

This principle is concerned with offering respect and protection to research participants through assurance of confidentiality of information shared and anonymity by not revealing the identity of individuals and institutions involved (Halai, 2006).

The respondents who participated in the study were requested to sign a consent form. The consent forms clearly stated that the information given was to be treated in strict confidentiality and no names or any form of identification was to be used that would link them with information provided. The consent form stated that participation was voluntary and that the interviewees could stop the interview at any moment. All the consent forms, interview sheets, and study material were kept in a safe and secure place. The researcher will destroy these materials when his supervisors instruct him to do so.

In general ethical principles were considered in all stages of the study. The participants were free to make decisions to answer or not, any questions. Secrecy was a priority and they did not disclose their names; only a code number in the questionnaire was used.

\section{Conclusion}

Pima CD4 is the first true point of care test for CD4. The Pima CD4 test consists of the Pima analyser and the Pima CD4 test cartridge.

The Pima analyser is a portable bench- top fixed volume cytometer used for the processing and analysis of a Pima test cartridge. For in vitro diagnostic use.

Pima CD4 provides an affordable, effective and valuable tool in the management of HIV patients.

Design to perform in laboratory as well as in non- Laboratory environments, the Pima analyser can be operated on external power or by using an on board rechargeable battery. The Pima analyser require no maintenance.

Results comes out within 20 minutes and the patient can wait. Capillary and venous blood can be used. Decentralized testing possible as Pima CD4 machine are now accessable in some health facilities. These allows the immediate measurement of CD4 counts, the further monitoring of the patients and as a consequence fast access to ART.

HIV patients are treated with antiretrovial therapy[ART] to suppress viral replication. Usually a mixture of several drugs is given. The initiation of treatment is determined by the concentration of $\mathrm{T}$ helper cells and the physical condition of the patient. However, there is need to increase the number of Pima CD4 machine to fasten the accessibility of treatment and diagnosis. 
Texila International Journal of Public Health

Volume 4, Issue 4, Dec 2016

\section{References}

[1] A. Cronje. Biomedical Science Research Methodology In Medical and Health Science- Nelson Mandela Metropolitan University. South Africa.

[2] Emmel N.(2013). Sampling and choosing cases in qualitative research; A realist approach London: Sage

[3] Gtlin LN. Lyons KJ. Successful grant writing. strategies for health and human service professionals. New York. Springer Publishing co.1996

[4] Kish, L.(1965). Survey Sampling.Wiley.ISBN0-471-48900-X.

[5] Medical Research Council of Canada. Guideline on research involving human subjects. Ottawa: Ministry of supply and services

[6] Marja J. Verhoef, PHD, Robert J. Hilsden, MD MSc FRCPC Departments of medicine and community Health Sciences University of Calgary, Alberta, Canada. 2001, 2004 RJ Hilsden, MJ Verlof.

[7] NIST/SEMATECH”7.2.4.2 Sample size required”, e Hand book of statistical methods

$[8]$

[9] Public Health Research methodology modules by Texila American University

[10] Pima analyser manual handbook

[11] San delowski, M.(1995). Sample size in qualitative research. Research in Nursing and Health,18,179-183

[12] Woodward DK. Clifton G.C. Development of successful research grant application. AM J hosp Pharm.51:813-822, 1994

[13] Zeiger M. Essentials of writing Biomedical Research papers. New York: McGraw-Hill Inch, 1991 